\title{
Adherence to the National Guidelines for Follow-Up Protocol in Subjects with Type 2 Diabetes Mellitus in Greece: The GLANCE Study
}

\author{
Nikolaos Papanas (D) - Moses Elisaf - Kalliopi Kotsa - Andreas Melidonis · \\ Stavros Bousboulas · Alexandra Bargiota - Emmanouel Pagkalos • \\ John Doupis · Ioannis Ioannidis · Iakovos Avramidis • \\ Angelos C. Pappas · Gerasimos Karousos • Eleni Arvaniti • \\ Magdalini Bristianou · Katerina Pietri · Eugenia Karamousouli • \\ Bernd Voss · Ilias Migdalis · Nikolaos Tentolouris
}

Received: July 22, 2020 / Accepted: September 19, 2020 / Published online: October 3, 2020

(c) The Author(s) 2020

\section{ABSTRACT}

Introduction: Physician adherence, or lack therefore, to diabetes care and follow-up guidelines may be linked to the rates of achieving suboptimal glycaemic, blood pressure and lipid targets in people with type 2 diabetes mellitus (T2DM). In this cross-sectional study

Digital Features To view digital features for this article go to https://doi.org/10.6084/m9.figshare.12967595.

Electronic supplementary material The online version of this article (https://doi.org/10.1007/s13300020-00935-6) contains supplementary material, which is available to authorized users.

\section{N. Papanas $(\square)$}

Diabetes Centre, Second Department of Internal Medicine, University General Hospital of Alexandroupolis, Democritus University of Thrace, Alexandroupolis, Greece

e-mail: papanasnikos@yahoo.gr

\section{Elisaf}

Department of Internal Medicine, School of Health Sciences, Faculty of Medicine, University of Ioannina, Ioannina, Greece

\section{K. Kotsa}

Division of Endocrinology and

Metabolism-Diabetes Centre, First Department of Internal Medicine, AHEPA University Hospital, Aristotle University of Thessaloniki School of Medicine, Thessaloniki, Greece we evaluated physician adherence to the patient follow-up protocol (PFP) of the 2017 Hellenic Diabetes Association (HDA) guidelines and also assessed glycated haemoglobin $\left(\mathrm{HbA}_{1 \mathrm{c}}\right)$, blood pressure and lipid control achievement rates in the routine care setting in Greece.

Methods: Eligible subjects were adults with T2DM receiving oral hypoglycaemic agents (OHAs) for $\geq 1$ year who had $\geq 2 \mathrm{HbA}_{1 \mathrm{c}}$ measurements in the previous year and an $\mathrm{HbA}_{1 \mathrm{c}}$ target $<7 \%$. Overall adherence at the subject level was defined as the percentage of the 62 HDA PFP items that had been met during the past year.
A. Melidonis
Diabetes Centre, General Hospital "Tzanio", Piraeus, Greece
S. Bousboulas
Diabetes Centre, General Hospital “Agios
Panteleimon", Piraeus, Greece
A. Bargiota Greece
E. Pagkalos
Diabetes Department, Clinic "Thermi",
Thessaloniki, Greece
Department of Endocrinology and Metabolic Diseases, University Hospital of Larissa, Thessaly, 
Results: Between June and December 2018, 601 eligible subjects $(54.6 \%$ men; mean age 65.2 years; median T2DM duration 5.9 years, of whom $96.5 \%$ had $\geq 1$ medical condition/comorbidity), were enrolled into the study by 53 hospital- and office-based endocrinologists, internists and general practitioners. The main OHAs prescribed at enrolment were metformin (91.0\%), dipeptidyl peptidase-4 inhibitors $(60.7 \%)$, sodium-glucose co-transporter-2 inhibitors (23.5\%) and sulphonylureas (16.3\%). Mean overall physician adherence to the PFP was $43.6 \%$. Predictors of greater higher physicans' adherence were female sex $(p=0.026),>3$ medical conditions/comorbidities $(p=0.043)$ and diabetic complications $(p<0.001)$. $\mathrm{HbA}_{1 \mathrm{c}}$, low-density lipoprotein-cholesterol, systolic/diastolic blood pressure and composite metabolic targets were achieved by $82.1,57.0,42.6$ and $21.6 \%$ of subjects, respectively.

Conclusions: In Greek routine care, physician adherence to the PFP of the 2017 HDA guidelines is suboptimal. Future efforts should focus on identifying the barriers to an adequate adherence by physicians to the full PFP, with the aim to provide optimal patient care.

Keywords: Follow-up; Glycated haemoglobin A1c; Guideline adherence; Target attainment; Type 2 diabetes mellitus

J. Doupis

Department of Internal Medicine and Diabetes, Salamis Naval and Veterans Hospital, Salamis Naval Base, Salamina, Greece

I. Ioannidis

First Department of Internal Medicine,

Konstantopouleio Hospital, Athens, Greece

I. Avramidis

Internal Medicine Department and Diabetes Centre, George Papanikolaou" General Hospital, Thessaloniki, Greece

A. C. Pappas

Diabetes Centre, Venizelio General Hospital

Heraklion, Heraklion, Crete, Greece

G. Karousos

Department of Internal Medicine and Diabetes, Athens Medical Group, Psychiko, Athens, Greece

\section{Key Summary Points}

Inadequate adherence to diabetes care and to follow-up guidelines by physicians may contribute to suboptimal glycaemic, lipid and blood pressure target attainment rates in subjects with type 2 diabetes (T2DM).

The aim of the GLANCE study was to assess the level of physician adherence to the patient follow-up protocol (PFP) of the Hellenic Diabetes Association (HAD) 2017 T2DM guidelines, the achievement rates for glycaemic, blood pressure and lowdensity lipoprotein-cholesterol (LDL-C) targets and physician adherence to the HDA-recommended pharmacological treatment algorithm.

Overall, physician adherence to the PFP of the national guidelines for T2DM was found to be suboptimal, but the glycaemic target was achieved by eight of ten subjects.

It is important to identify the barriers to an adequate adherence by physicians to the full PFP provided in the 2017 HDA guidelines, with the aim to optimally support subjects with T2DM and improve outcomes.

E. Arvaniti

General Hospital of Ioannina "G. Hatzikosta", Ioannina, Greece

M. Bristianou

Department of Internal Medicine, General Hospital of Lamia, Lamia, Greece

K. Pietri · E. Karamousouli

Merck Sharp and Dohme (MSD), A1, Athens, Greece

B. Voss

Merck Sharp and Dohme (MSD) RBSC GmbH, Haar, Germany

I. Migdalis

Second Medical Department and Diabetes Centre, NIMTS Hospital, Athens, Greece 


\section{DIGITAL FEATURES}

This article is published with digital features to facilitate understanding of the article. You can access the digital features on the article's associated Figshare page. To view digital features for this article go to https://doi.org/10.6084/m9. figshare.12967595.

\section{INTRODUCTION}

Diabetes is an emerging global epidemic of the twenty-first century, with an ever-increasing prevalence [1]. The International Diabetes Federation (IDF) 2019 estimate of diabetes prevalence in Greece was $7.4 \%$ of the national population [1]. Similarly, a study based on 2014-2015 Greek national electronic prescription database data estimated the prevalence of medication-prescribed diabetes at $8.2 \%$ for adults in general and at $30.3 \%$ for those aged $\geq$ 75 years [2].

Type 2 diabetes (T2DM) accounts for about $90 \%$ of all cases of diabetes [3]. Persons with T2DM carry a high risk of developing microand macrovascular complications, with atherosclerotic cardiovascular disease (CVD) being the primary source of morbidity and mortality [4-6]. Current management goals include achieving adequate glycaemic control and addressing vascular risk factors, notably blood pressure (BP), lipids, among others [7-10]. In general, the achievement of therapeutic targets in T2DM is not optimal $[11,12]$; this also holds true for Greece [13-16], where the achievement rates of glycated haemoglobin $\left(\mathrm{HbA}_{1 \mathrm{c}}\right)$ and $\mathrm{BP}$ targets have been reported to be 53 and $27 \%$, respectively [15]. Achievement of therapeutic targets may be linked to adherence to diabetes standards of medical care recommendations [17-19]. In Greece, the Hellenic Diabetes Association (HDA) issues and regularly updates practice guidelines for the management

\section{N. Tentolouris}

First Department of Propaedeutic Internal Medicine, Laiko General Hospital, National Kapodistrian University of Athens Medical School, Athens, Greece and care of persons with diabetes [20], which are aligned with the guidelines of the American Diabetes Association (ADA) and European Association for the Study of Diabetes (EASD) [21-23]. The 2017 HDA guidelines include a patient follow-up protocol (PFP) that lists 62 items that should be part of a patient's medical history and clinical and laboratory evaluations, with the aim to achieve optimal diabetes management [20].

To date, the adherence of Greek physicians to the PFP has not been systematically explored. The aims of this study were to assess (1) the adherence level of physicians to the PFP of the HDA 2017 T2DM guidelines [20] and (2) the achievement rates of glycaemic, BP and lowdensity lipoprotein-cholesterol (LDL-C) control; and (3) to examine physician adherence to the HDA-recommended pharmacological treatment algorithm.

\section{METHODS}

\section{Study Design}

The present study (GLANCE) was a nationwide, multi-centre, cross-sectional study conducted in 15 hospital-based outpatient clinics and 38 private practices located across Greece. A total of 610 persons with T2DM were consecutively enrolled in the study (referred to as subjects) by 14 endocrinologists, 35 internists and four general practitioners with a special interest in diabetes between 27 June and 20 December 2018. The study was conducted in accordance with the International Society for Pharmacoepidemiology guidelines for Good Pharmacoepidemiology Practice, the ethical principles of the Declaration of Helsinki of 1964 and its later amendments and all standing regulations. The study was approved by the ethics committees of all participating hospitals (see Electronic Supplementary Material Table S1). Signed written informed consent was obtained from all participants.

The study included non-insulin-dependent persons with T2DM who were aged $\geq 19$ years at enrolment, had $\geq 2 \mathrm{HbA}_{1 \mathrm{c}}$ measurements during the past year, had a $\mathrm{HbA}_{1 \mathrm{c}}$ target $<7 \%$ and 
were receiving oral hypoglycaemic agents (OHAs) for $\geq 1$ year. Exclusion criteria were patients who were insulin dependent; had type $1 \mathrm{DM}$ or gestational diabetes; were hospitalised; were participating in any clinical trial at time of enrolment; had in the current or past (preceding) year a history of alcohol or drug abuse; were pregnant/lactating (at enrolment or within the previous year); subjects who did not have $\mathrm{HbA}_{1 \mathrm{c}}<7 \%$ as a therapeutic target were also excluded.

In order to ensure country-wide representation of clinical practices, the sample of patients was distributed according to the percentage of the Greek population residing in the various regions. Consecutive patients from those attending the selected centres during a prespecified period were invited to participate in the study.

Data were collected using a web-based data system during the single study visit, which occurred at enrolment, and through patient self-report and medical chart review. For the evaluation of adherence by physicians to the HDA guidelines, we used information available in participants' medical records within the last 365 days and up tol the most recent visit prior to enrolment. For the assessment of metabolic target attainment rates, we used laboratory assessments available at enrolment or within 1 month prior to enrolment. Compliance of participants to their current antidiabetic treatment regimen and their adherence to physician-provided nutritional recommendations were rated by the physicians using a five-level Likert-type scale with responses of "very good", "good", "moderate", "poor" and "very poor".

\section{Outcomes}

The primary outcome was overall physican adherence to the PFP of the HDA guidelines, at a participant level. Other outcomes included: (1) glycaemic control, defined as the proportion of subjects attaining the $\mathrm{HbA}_{1 \mathrm{c}}$ target $<7 \%$; (2) adherence to the HDA-recommended therapeutic algorithm; (3) physician adherence to each domain and each item of the PFP; (4) adherence to the PFP according to each physician's specialty (endocrinologist, internist, general practitioner); and (5) target achievement for LDL-C, BP and composite metabolic control, in accordance with the HDA T2DM guidelines [20], as follows: (1) systolic BP (SBP) $<140 \mathrm{mmHg} \quad(<130 \mathrm{mmHg}$ for subjects $<65$ years old) and diastolic BP (DBP) $<85 \mathrm{mmHg} \quad(<80 \mathrm{mmHg}$ for subjects $<65$ years old); (2) composite SBP/DBP target of SBP $<140$ and $\mathrm{DBP}<85 \mathrm{mmHg}$ for subjects aged $\geq 65$ years old, and $\mathrm{SBP}<130$ and DBP $<80 \mathrm{mmHg}$ for those aged $<65$ years; (3) $\mathrm{LDL}-\mathrm{C}<100 \mathrm{mg} / \mathrm{dL}$ or $<70 \mathrm{mg} / \mathrm{dL}$ in subjects with pre-existing CVD (i.e. coronary artery disease, peripheral arterial disease, stroke, transient ischaemic attack, carotid artery stenosis (> 50\%) and abdominal aortic aneurysm); (4) composite metabolic control, i.e. simultaneous attainment of $\mathrm{HbA}_{1 \mathrm{c}}$, LDL-C, SBP and DBP targets as defined above.

\section{Follow-Up Protocol}

The PFP of the HDA 2017 guidelines [20] comprises 62 items (Table 1), organised in three distinct domains: complete medical history (items 1-30), physical examination (items 31-42) and laboratory evaluation (items 43-62). For the following items to be considered to be completed, the respective information should have been available in the patient's medical record: items 1, 3-10, 13, 21-29, 31-37, 40, 43 on every visit, and items $44-58$ and 62 at least once in the year prior to enrolment. The remaining items were considered fulfilled if the physician confirmed they had been evaluated.

\section{Statistical Analyses}

Statistical analyses were performed using SAS ${ }^{\circledR}$ software version 9.4 (SAS Institute Inc., Cary, NC, USA). Categorical variables are presented as frequencies $(n, \%)$, while continuous variables are presented as the mean and standard deviation (SD) or as the median and interquartile range (IQR). Normality of distribution was assessed using the Kolmogorov-Smirnov test. For each subject, a crude score of overall adherence was estimated as the sum of fulfilled 
Table 1 Items and domains based on the patient's follow-up protocol of the Hellenic Diabetes Association guidelines and physicians' adherence to each item

\begin{tabular}{|c|c|c|}
\hline $\begin{array}{l}\text { Item } \\
\text { no. }\end{array}$ & Domains of the 2017 HDA guidelines & $\begin{array}{l}\text { Patients for whom sufficient information } \\
\text { was available for the item }[n(\%)]\end{array}$ \\
\hline
\end{tabular}

Complete medical history domain

A) Demographic characteristics

$1 \quad$ Age (or date of birth)

$589(98.0)$

2 Profession

$471(78.4)$

3 Year of T2DM diagnosis

$560(93.2)$

$4 \quad$ Mode of T2DM diagnosis

$297(49.4)$

5 Medication applied to T2DM since diagnosis

$503(83.7)$

6 Smoking status (no. of cigarettes, etc.)

$63(10.5)$

7 Alcohol consumption

$59(9.8)$

$8 \quad$ Use of toxic substances

$172(28.6)$

9 Vaccinations

$96(16.0)$

B) Factors that should have been recorded on the last visit prior to enrolment

10 Present symptoms

$181(30.1)$

11 Current antidiabetic treatment

$601(100.0)$

12 Response to treatment

$564(93.8)$

13 Other medications received (concomitant medication)

$214(35.6)$

14 Dietary habits

$405(67.4)$

15 Diet to be followed

$341(56.7)$

16 Nutrition state

$314(52.2)$

17 Physical activity

$375(62.4)$

18 Patient's compliance with medication intake

$422(70.2)$

19 Willingness for dietary and lifestyle modifications

$299(49.8)$

C) Information that the patient has to provide

20 Blood sugar self-measurement results

$331(55.1)$

21 Hypoglycaemic events

$123(20.5)$

22 Ketoacidosis events (Incidence, severity/cause)

$60(10.0)$

23 Diabetic complications (time and method of diagnosis)

$73(12.1)$

D) Other clinical history information

24 Hypertension

$119(19.8)$

25 Dyslipidaemia

$119(19.8)$ 
Table 1 continued

Item Domains of the 2017 HDA guidelines

no.

26 Body weight changes over time

27 Concomitant diseases (e.g., depression, obstructive sleep apnoea, non-alcoholic liver disease, osteoporosis, periodontal disease, neoplasms)

28 Psychosocial problems

29 Surgical procedures

E) Family history

30 Diabetes mellitus presence in parents, siblings or children

Physical examination domain

$141(23.5)$

$110(18.3)$

$42(7.0)$

$514(85.5)$

31 Clinical examination relates to all systems (respiratory,

Patients for whom sufficient information was available for the item [ $n(\%)]$

A) At the first visit and repeated on a yearly basis cardiovascular, digestive, urinary, nervous system), skin, skull, muscles, joints, sensory organs

32 Measurement of height, body weight, BMI calculation

33 Waist circumference measurement

34 Pulse and arterial pressure measurement (systolic and diastolic, in a seated and standing position, as well as assessment of the difference in blood pressure measurements between the two upper extremities)

35 Lower-extremity examination for the diagnosis of diabetic peripheral neuropathy (foot architecture, Achilles reflexes, examination of small- and large nerve fibre sensation)

36 Examination of the lower extremities for the diagnosis of peripheral arterial disease (peripheral arteries pulses, ankle/ brachial index, skin colour and temperature, examination of hair distribution and state of nails and examination for: oedema, sensitivity, pain, ulcers, gangrene, infection, bubbles, abrasions, skin ruptures, bunions, keratoses)

37 Examination for the diagnosis of autonomous nervous system $66(11.0)$ neuropathy (gustatory sweating, persistent tachycardia at rest, orthostatic hypotension, hypoglycaemia unawareness, sexual dysfunction)

38 Thyroid gland palpation $211(35.1)$ 
Table 1 continued

Item Domains of the $2017 \mathrm{HDA}$ guidelines

no.

39 Skin examination: Search of acanthosis nigricans and possible

hypertrophy/atrophy at the sites of insulin injection

Ophthalmology examination

$40 \quad$ Fundoscopy upon dilation at diagnosis

$218(36.3)$

41 If, during the first fundoscopy, there are no findings, this is being

$201(33.4)$ repeated after 1 year, and then every year

42 If there are lesions, the examination is performed every six months

$328(54.6)$ or more frequently depending on the severity of the lesions

Laboratory evaluation

A) At every visit

43 Glucose measurement, either at a fasting state or $2 \mathrm{~h}$ after the meal $258(42.9)$ or randomly, depending on the time of the visit

B) Every 3-6 months (at least once within the year prior to enrolment)

$44 \quad \mathrm{HbA}_{1 \mathrm{c}} \%$ measurement

$601(100.0)$

C) Every year (At least once within the year prior to enrolment)

45 Complete blood count, ESR

$96(16.0)$

46 Total cholesterol

$427(71.0)$

47 Triglycerides

432 (71.9)

48 HDL-C

$420(69.9)$

49 LDL-C

$50 \quad$ Urea

$412(68.6)$

51 Creatinine

$373(62.1)$

52 Potassium

$432(71.9)$

$253(42.1)$

53 Sodium

$231(38.4)$

54 Glomerular filtration calculation (eGFR) as per MDRD or CKD- 116 (19.3) EPI

55 Aminotransferases (AST, ALT)

$374(62.2)$

56 Alkaline phosphatase

$184(30.6)$

$57 \quad \gamma \mathrm{GT}$

$234(38.9)$

$58 \quad \mathrm{CPK}$

$157(26.1)$

59 Thyroid function (TSH): in patients with dyslipidemia and in $395(65.7)$

women aged $>50$ years 
Table 1 continued

\begin{tabular}{lll}
\hline $\begin{array}{l}\text { Item } \\
\text { no. }\end{array}$ & Domains of the 2017 HDA guidelines & $\begin{array}{l}\text { Patients for whom sufficient information } \\
\text { was available for the item }[\boldsymbol{n}(\%)]\end{array}$ \\
\hline 60 & $\begin{array}{l}\text { Urine examination (if albuminuria is determined, examination of } \\
\text { its origin) }\end{array}$ & $246(40.9)$ \\
61 & $\begin{array}{l}\text { Examination for urinary albumin excretion by calculating the } \\
\text { albumin/creatinine ratio at a random sample of morning urine }\end{array}$ & $102(17.0)$ \\
62 & ECG at rest & $59(9.8)$ \\
\hline
\end{tabular}

$A S T$ Aspartate transaminase, $A L T$ alanine transaminase, CKD-EPI Chronic Kidney Disease Epidemiology Collaboration, $C P K$ creatine phosphokinase, $\gamma G T$ gamma-glutamyl-transferase, $E C G$ electrocardiogram, $e G F R$ estimated glomerular filtration rate, ESR erythrocyte sedimentation rate, $H b A 1 c$ haemoglobin Alc, $H D A$ Hellenic Diabetes Association, $H D L-C$ high-density lipoprotein cholesterol, $L D L-C$ low-density lipoprotein cholesterol, MDRD Modification of Diet in Renal Disease, T2DM type 2 diabetes mellitus, TSH thyroid stimulating hormone

items, divided by the total number of PFP items $(n=62)$, multiplied by 100 . Each item was scored 1 point if fulfilled or 0 if not. The mean overall adherence 95\% confidence interval (CI) was calculated. The 95\% Wald CI for binomial proportions is presented for the glycaemic control rate.

To identify patient- and physician-related factors associated with physicians' adherence to the HDA guidelines PFP, we performed univariable and multivariable linear regression analyses. The initial multivariable model included the following factors: participant's age at enrolment ( $\geq 65$ vs. $<65$ years), sex, T2DM duration ( $>5$ vs. $\leq 5$ years), obesity, number of past or ongoing medical conditions/comorbidities ( $>3$ vs. $\leq 3$ ), presence of diabetic complications and physicians' specialty (endocrinologist vs. general practitioner, internist vs. general practitioner). The final multivariable model was derived through a stepwise procedure based on the minimisation of the Akaike information criterion.

\section{Sample Size}

Due to the descriptive nature of the study, sample size calculation was based on the study's primary endpoint, and no hypothesis-specific calculation was performed. Assuming that the estimated SD of the overall adherence score could range from 0.5 to approximately 1 , a sample size of 600 participants was considered appropriate to estimate the adherence level with a 95\% CI halfwidth $<0.1$, with at least $80 \%$ power.

\section{RESULTS}

\section{Baseline Characteristics at Enrolment}

A total of 610 subjects were enrolled. Of these, 601 [mean (SD); age 65.2 (10.3) years; $54.6 \%$ men] were included in the analysis (Fig. 1). Participants' demographic and anthropometric characteristics are presented in Table 2. Mean (SD) age at T2DM diagnosis was 57.3 (10.4) years, with a median disease duration of 5.9 years (Table 2). Median (IQR) subject followup duration was 2.6 (1.5-4.7) years, with $29.5 \%$ of subjects having been diagnosed with T2DM by the study physicians.

Overall, $96.5 \%$ of participants had $\geq 1$ [median (IQR): 3.0 (2.0-4.0)] clinically relevant medical condition/comorbidity. Conditions/comorbidities reported in $\geq 10 \%$ of the participants are shown in Table 2. At enrolment, 93.8\% (563/600) of subjects were receiving at least one concomitant medication other than antidiabetics. Medication classes received by $>10 \%$ of the participants included lipidmodifying agents $(73.5 \%)$, cardiac therapy/antihypertensives $(66.5 \%)$ and antithrombotic or antiplatelet agents (30.5\%). 


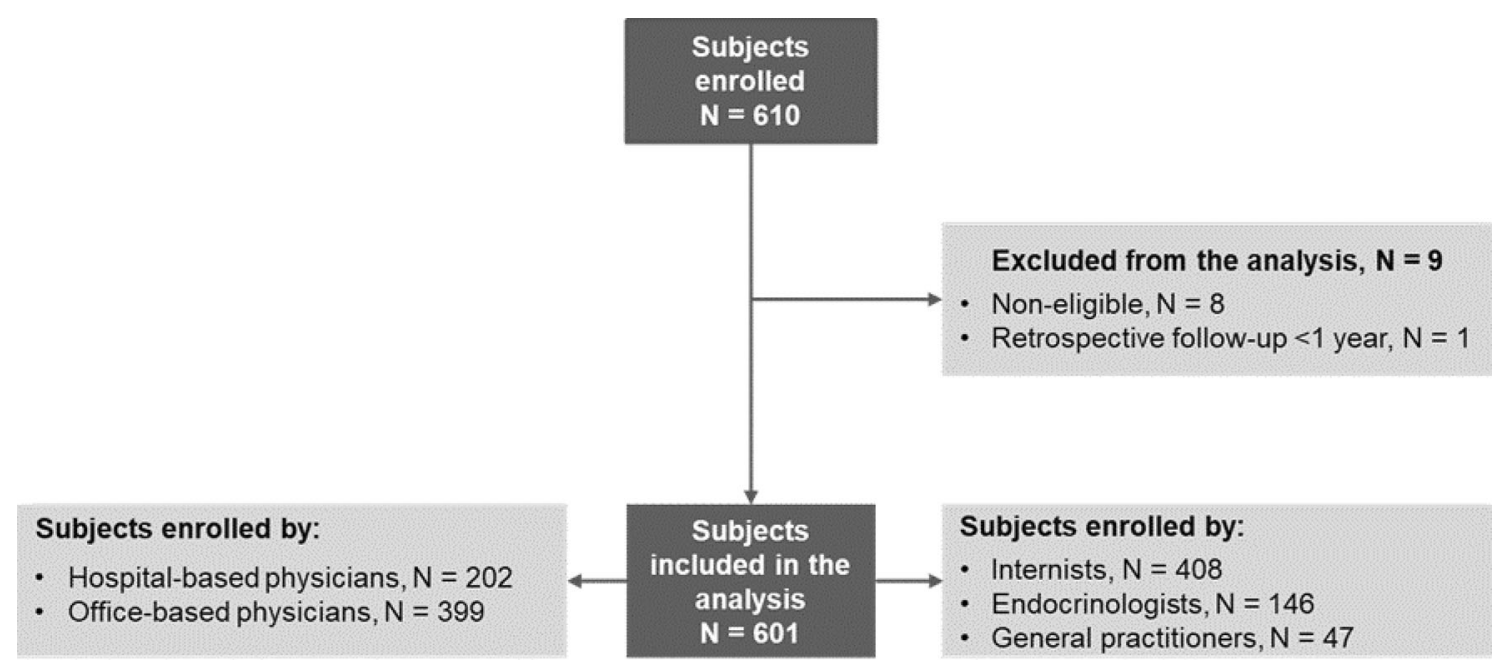

Fig. 1 Subject disposition and flow chart. Flow chart displaying the number of subjects enrolled and analysed, and the number of enrolled subjects analysed per physician specialty and healthcare setting

Frequency of diabetic complications was $16.1 \%: 6.5 \%$ of the subjects had macrovascular, $6.0 \%$ microvascular and $7.0 \%$ other diabetic complications (Table 3). Additionally, 12 months prior to enrolment, $4.4 \%$ of the 571 evaluable subjects had experienced a total of 134 (median 3) hypoglycaemic events. The latter included 75 of unknown severity and 59 mild ones. Prior to enrolment, most subjects had received training on diabetes self-management and personalised nutrition counselling, and most were using a self-monitoring glucose device (Table 3).

\section{Treatment for T2DM and Subjects' Compliance to Medication}

Antidiabetic therapy since T2DM diagnosis was known for 509 (84.7\%) of subjects. Among these, $30.5 \%$ had received one, $31.2 \%$ two and $38.3 \% \geq 3$ antidiabetic agents (Table 4). A median (IQR) of 4.0 (2.1-9.0) years had elapsed from first receipt of an OHA to enrolment.

At enrolment, all subjects were receiving OHAs with a median (IQR) treatment duration of $3.8(2.0-8.9)$ years. Specifically, $27.6 \%$ of subjects were receiving metformin monotherapy, $63.4 \%$ were receiving metformin + other OHAs and $9.0 \%$ were receiving therapy that did not include metformin (Table 4). Participants' compliance with antidiabetic medication was rated by the physicians on a five-level scale: $67.5 \%(400 / 593)$ of the subjects were rated as having very good compliance, $26.0 \%(154 / 593)$ as good, $3.9 \%(23 / 593)$ as moderate and $2.7 \%$ $(16 / 593)$ as poor or very poor. In addition, compliance was rated very good/good in $95.4 \%$ $(188 / 197)$ of those receiving monotherapy, in $95.4 \%(208 / 218)$ of those on dual therapy, in $88.8 \%(135 / 152)$ of those receiving triple therapy and in $92.0 \%(23 / 25)$ of those on quadruple therapy.

\section{Guidelines Followed by Physicians}

Physicians followed the HDA therapeutic guidelines for managing $77.2 \%$ of the subjects, the ADA/EASD guidelines for $43.4 \%$ and the American Association of Clinical Endocrinologists/American College of Endocrinology (AACE/ACE) guidelines for $8.8 \%$, while different guidelines were followed according to physicians' specialty (Fig. 2). Hospital-based and office-based physicians followed the HDA guidelines for managing $85.6 \%(173 / 202)$ and $72.9 \%(291 / 399)$ of the subjects, the ADA/EASD guidelines for $40.1 \%(81 / 202)$ and $45.1 \%$ (180/ 399 ), and the AACE/ACE guidelines for $12.4 \%$ $(25 / 202)$ and $7.0 \%(28 / 399)$, respectively. 
Table 3 Type 2 diabetes history, training on diabetes selfmanagement, nutrition counselling and healthcare resource utilisation in the year prior to enrolment

\begin{tabular}{ll}
\hline Clinical aspects of subjects & Values \\
\hline Characteristics of patient history of T2DM & \\
Age at T2DM diagnosis [mean (SD), & $57.3(10.4)$ \\
years], $N=591$ & \\
Duration of T2DM [median (IQR), & 5.9 \\
years], $N=591$ & $(2.9-11.7)$ \\
Family history of diabetes mellitus, & $323(63.2 \%)$ \\
$N=511$ & \\
Number of HbA 1 assessments during the & 2.0 \\
last year prior to enrolment [median & $(2.0-3.0)$ \\
(IQR)], $N=601$ & \\
Diabetes complications, $N=601$ & $97(16.1 \%)$ \\
Macrovascular complications & $39(6.5 \%)$ \\
Coronary artery disease & $28(4.7 \%)$ \\
Peripheral arterial disease & $8(1.3 \%)$ \\
Transient ischaemic attack & $3(0.5 \%)$ \\
Cerebrovascular event & $2(0.3 \%)$ \\
Cardiac failure & $1(0.2 \%)$ \\
Carotid artery stenosis $(>50 \%)$ & $1(0.2 \%)$ \\
Microvascular complications & $36(6.0 \%)$ \\
Peripheral neuropathy & $14(2.3 \%)$ \\
Chronic kidney disease & $10(1.7 \%)$ \\
Autonomic neuropathy & $9(1.5 \%)$ \\
Retinopathy & $9(1.5 \%)$ \\
Diabetic foot & $2(0.3 \%)$ \\
Erectile dysfunction & $2(0.3 \%)$ \\
Other diabetic complications & $42(7.0 \%)$ \\
Pats' &
\end{tabular}

Patients' training and blood glucose monitoring

Training on diabetes self-management $536(89.2 \%)$

(during the last year prior to enrolment), $N=601$

Personalised nutrition counselling for $549(91.3 \%)$ achieving glycaemic control, $N=601$
Table 3 continued

\begin{tabular}{lc}
\hline Clinical aspects of subjects & Values \\
\hline $\begin{array}{l}\text { Patients' adherence to nutrition recommendations as } \\
\text { assessed by the physician, } N=529\end{array}$ \\
$\begin{array}{l}\text { Very good/good } \\
\text { Moderate }\end{array}$ & $163(59.0 \%)$ \\
Poor/very poor & $54(10.2 \%)$ \\
Current use of blood glucose self- & $527(87.7 \%)$ \\
monitoring device, $N=601$ & $522(86.9 \%)$ \\
Current use of blood glucose self- \\
monitoring device for at least 1 year prior \\
to enrolment, $N=601$ \\
$\begin{array}{l}\text { Current use of continuous blood glucose } \\
\text { monitoring system, } N=601\end{array}$ \\
$\begin{array}{l}\text { Healthcare resource utilisation in the } 12 \text { months prior to } \\
\text { enrolment, } N=601\end{array}$ \\
$\begin{array}{l}\text { Total number of visits to study physicians' } \\
\text { clinic/office }\end{array}$ \\
$\begin{array}{l}\text { Number of visits [median (IQR)] } \\
\end{array}$ \\
\hline
\end{tabular}

Values in table are given as a frequency (number with percentage in parentheses), unless otherwise indicated $N$ Number of subjects for whom information was available

evaluation domain (mean 48.3\%, 95\% CI 46.2-50.3), followed by the complete medical history domain (mean 45.5\%, 95\% CI 43.9-47.1) and then by the physical examination domain (mean 31.2\%, 95\% CI 29.3-33.2) (Fig. 2).

Mean overall adherence and the results for adherence to each PFP domain according to physicians' specialty are presented in Fig. 2. The percentage of subjects for whom each of the 62 PFP items was completed are shown in Table 1. Items completed in $>90 \%$ of subjects included the items "current antidiabetic treatment", "age or date of birth", "response to treatment", "year of T2DM diagnosis" and "HbA $\mathrm{H}_{1 \mathrm{c}}$ measurement". Items fulfilled in $<10 \%$ of the subjects were the 
Table 4 Medications used by study subjects for management of type 2 diabetes mellitus prior to study enrolment and at enrolment

\begin{tabular}{ll}
\hline Medications & $\begin{array}{l}\text { Number of } \\
\text { subjects (\%) }\end{array}$ \\
\hline
\end{tabular}

Antidiabetic treatment prior to study enrolment

Subjects for whom the treatment classes 509 (84.7) received prior to enrolment were fully

known, $N=601$

Treatment classes, $N=509$

Biguanides/metformin

$495(97.2)$

DPP4i

SGLT2i

$130(25.5)$

Sulphonylureas

Glitazones

Meglitinides

Insulin

$4(0.8)$

GLP-1 analogues

$3(0.6)$

$2(0.4)$

Alpha-glucosidase inhibitors

Combinations of prior drug classes, $N=509$

Metformin only

$148(29.1)$

Metformin and DPP4i

$121(23.8)$

Metformin, DPP4i and SGLT2i

$56(11.0)$

Metformin, sulphonylureas and DPP4i

$51(10.0)$

Other combinations of drug classes

$119(23.4)$

including metformin

Combinations not including metformin

$14(2.8)$

Antidiabetic treatment at enrolment

Any treatment, $N=601$

$601(100.0)$

Treatment classes, $N=601$

Biguanides/metformin

DPP4i

$365(60.7)$

SGLT2i

$141(23.5)$

Sulphonylureas

$98(16.3)$

Glitazones

$57(9.5)$

Meglitinides

$3(0.5)$
Table 4 continued

\begin{tabular}{ll}
\hline Medications & $\begin{array}{l}\text { Number of } \\
\text { subjects (\%) }\end{array}$ \\
\hline Number of drug classes, $N=601$ & \\
1 & $198(32.9)$ \\
2 & $223(37.1)$ \\
3 & $154(25.6)$ \\
4 & $25(4.2)$ \\
5 & $1(0.2)$ \\
Patterns of drug classes, $N=601$ & \\
Metformin only & $166(27.6)$ \\
Metformin + DPP4i & $164(27.3)$ \\
Metformin + DPP4i + SGLT2i & $72(12.0)$ \\
Metformin + Sslphonylureas + DPP4i & $42(7.0)$ \\
Metformin + other & $103(17.1)$ \\
Combinations not including metformin & $54(9.0)$ \\
\hline
\end{tabular}

$D P P 4 i$ Dipeptidyl peptidase- 4 inhibitors, GLP- 1 glucagonlike peptide-1, $N$ number of subjects for whom information was available, $S G L T 2 i$ sodium-glucose co-transporter2 inhibitors

items "alcohol consumption", "psychosocial problems", "surgical procedures" and "electrocardiogram at rest'.

The overall adherence of physicians to the PFP was higher for female subjects $(p=0.026)$, subjects with $>3$ medical conditions/comorbidities $(p=0.043)$, those with diabetic complications $(p<0.001)$ and those seen by endocrinologists versus general practitioners $(p<0.001)$ and by internists versus general practitioners $(p=0.001)$, when adjusted for other factors in the final multivariable model (Table 5). Significant predictors of physicians' adherence overall and to each of the PFP domains are shown in Table 5. 


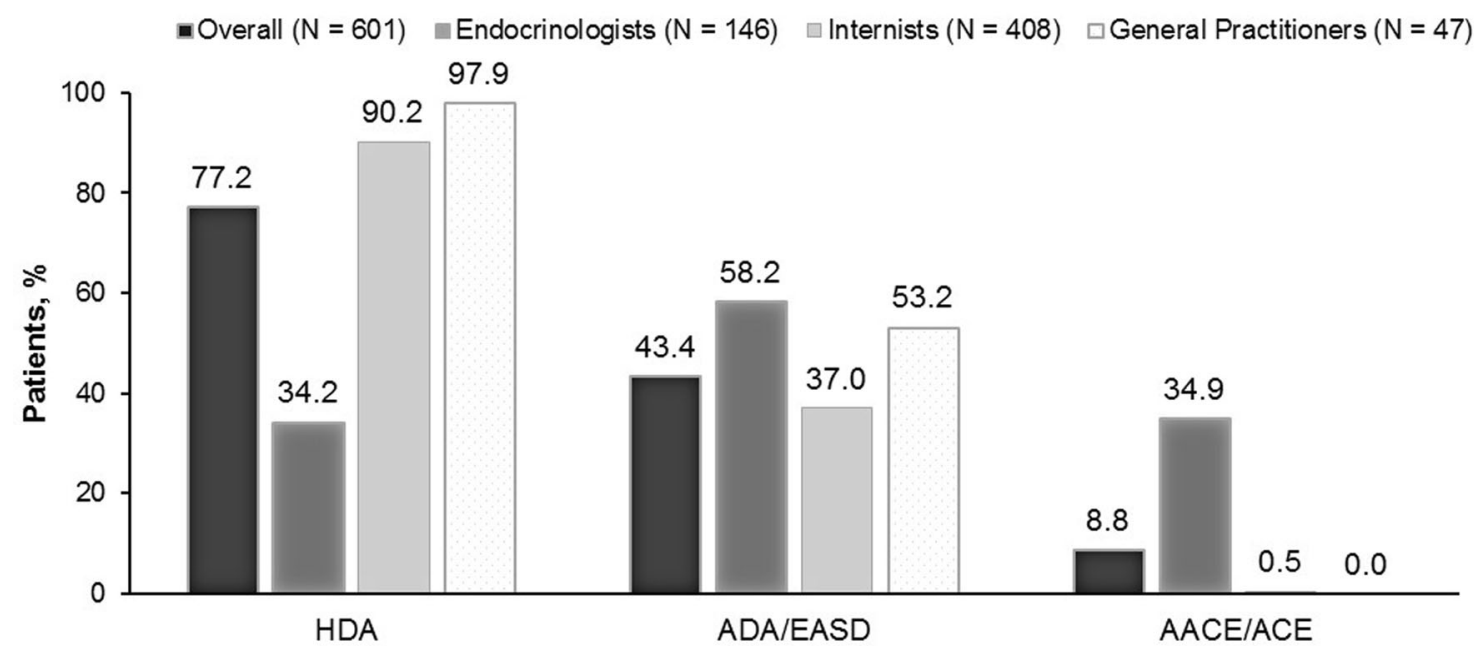

Fig. 2 Diabetes guidelines followed by physicians overall and according to physicians' specialty. Bars and labels indicate percentage of subjects for which each guideline was followed. More than one guideline was followed for some participants. AACE/ACE American Association of
Clinical Endocrinologists/American College of Endocrinology, ADA/EASD American Diabetes Association/European Association for the Study of Diabetes, $H D A$ Hellenic Diabetes Association

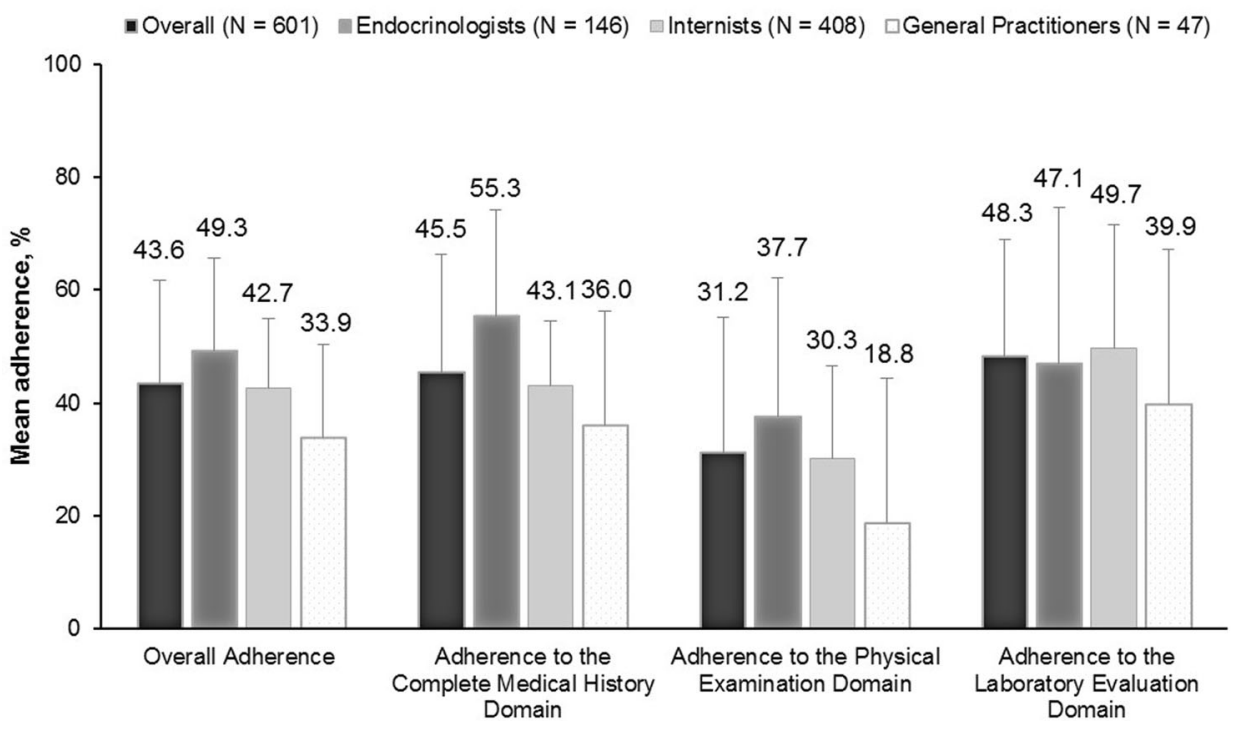

Fig. 3 Adherence to the patient follow-up protocol (PFP) of the HDA guidelines overall and according to physicians' specialty. Bars represent the mean and standard deviation, while the labels indicate means

\section{Achievement of Glycaemic Control at Enrolment}

Based on available $\mathrm{HbA}_{1 \mathrm{c}}$ measurements, adequate glycaemic control $\left(\mathrm{HbA}_{1 \mathrm{c}}<7 \%\right)$ was achieved by $82.1 \%$ of all participants (363/442, 95\% CI 78.6-85.7; Fig. 4a). Among the 79 $(17.9 \%)$ subjects without adequate glycemic control, 71 had $\mathrm{HbA}_{1 \mathrm{c}} 7-8.5 \%$, one had $\mathrm{HbA}_{1 \mathrm{c}}>8.5$ and $\leq 9 \%$, and seven had 
Table 5 Predictors of physicians' adherence to the type 2 diabetes mellitus patient follow-up protocol (PFP) of the Hellenic Diabetes Association guidelines (multivariable linear regression), overall and for each PFP domain

\begin{tabular}{|c|c|c|c|c|c|c|}
\hline \multirow{2}{*}{$\begin{array}{l}\text { Adherence to PFP overall and for each } \\
\text { PFP domain }\end{array}$} & \multirow{2}{*}{ Comparison } & \multirow{2}{*}{$\begin{array}{l}\text { OLS } \\
\text { estimate }\end{array}$} & \multirow{2}{*}{$\begin{array}{l}\text { Standard } \\
\text { error }\end{array}$} & \multicolumn{2}{|c|}{ 95\% Wald CI } & \multirow[t]{2}{*}{$p$ value } \\
\hline & & & & $\begin{array}{l}\text { Lower } \\
\text { limit }\end{array}$ & $\begin{array}{l}\text { Upper } \\
\text { limit }\end{array}$ & \\
\hline \multicolumn{7}{|l|}{ Overall adherence $(N=526)$} \\
\hline Sex & Male vs. female & -3.163 & 1.417 & -5.947 & -0.380 & $0.026^{*}$ \\
\hline Duration of T2DM & $\begin{array}{l}>5 \\
\text { vs. } \leq 5 \text { years }\end{array}$ & 1.819 & 1.427 & -0.985 & 4.623 & 0.203 \\
\hline $\begin{array}{l}\text { Number of medical } \\
\text { conditions/comorbidities }\end{array}$ & $>3$ vs. $\leq 3$ & 3.342 & 1.644 & 0.112 & 6.571 & $0.043^{*}$ \\
\hline Diabetic complications & Yes vs. no & 7.929 & 1.941 & 4.116 & 11.742 & $<0.001^{*}$ \\
\hline \multirow[t]{2}{*}{ Physicians' specialty } & $\begin{array}{l}\text { Endocrinologist } \\
\text { vs. GP }\end{array}$ & 16.062 & 3.072 & 10.027 & 22.097 & $<0.001^{*}$ \\
\hline & Internist vs. GP & 9.083 & 2.831 & 3.521 & 14.645 & $0.001^{*}$ \\
\hline \multicolumn{7}{|l|}{ Complete medical history domain $(N=536)$} \\
\hline Sex & Male vs. female & -3.482 & 1.616 & -6.658 & -0.307 & $0.032^{*}$ \\
\hline Diabetic complications & Yes vs. no & 10.935 & 2.106 & 6.797 & 15.072 & $<0.001^{*}$ \\
\hline \multirow[t]{2}{*}{ Physicians' specialty } & $\begin{array}{l}\text { Endocrinologist } \\
\text { vs. GP }\end{array}$ & 22.684 & 3.390 & 16.025 & 29.342 & $<0.001^{*}$ \\
\hline & Internist vs. GP & 10.060 & 3.137 & 3.898 & 16.221 & $0.001^{*}$ \\
\hline \multicolumn{7}{|l|}{ Physical examination $(N=591)$} \\
\hline Sex & Male vs. female & -4.072 & 1.935 & -7.871 & -0.272 & $0.036^{*}$ \\
\hline Duration of $\mathrm{T} 2 \mathrm{DM}$ & $\begin{array}{l}>5 \\
\text { vs. } \leq 5 \text { years }\end{array}$ & 3.029 & 1.958 & -0.815 & 6.874 & 0.122 \\
\hline $\begin{array}{l}\text { Number of medical } \\
\text { conditions/comorbidities }\end{array}$ & $>3$ vs. $\leq 3$ & 8.714 & 2.097 & 4.596 & 12.833 & $<0.001^{*}$ \\
\hline \multirow[t]{2}{*}{ Physicians' specialty } & $\begin{array}{l}\text { Endocrinologist } \\
\text { vs. GP }\end{array}$ & 16.525 & 4.052 & 8.566 & 24.484 & $<0.001^{*}$ \\
\hline & Internist vs. GP & 9.174 & 3.721 & 1.867 & 16.482 & $0.014^{*}$ \\
\hline \multicolumn{7}{|l|}{ Laboratory evaluation $(N=591)$} \\
\hline Age at enrolment & $\begin{array}{l}\geq 65 \\
\text { vs. }<65 \text { years }\end{array}$ & 4.020 & 2.185 & -0.271 & 8.312 & 0.066 \\
\hline Duration of $\mathrm{T} 2 \mathrm{DM}$ & $\begin{array}{l}>5 \\
\text { vs. } \leq 5 \text { years }\end{array}$ & 3.882 & 2.181 & -0.401 & 8.164 & 0.076 \\
\hline
\end{tabular}


Table 5 continued

\begin{tabular}{|c|c|c|c|c|c|c|}
\hline \multirow{2}{*}{$\begin{array}{l}\text { Adherence to PFP overall and for each } \\
\text { PFP domain }\end{array}$} & \multirow{2}{*}{ Comparison } & \multirow{2}{*}{$\begin{array}{l}\text { OLS } \\
\text { estimate }\end{array}$} & \multirow{2}{*}{$\begin{array}{l}\text { Standard } \\
\text { error }\end{array}$} & \multicolumn{2}{|c|}{ 95\% Wald CI } & \multirow[t]{2}{*}{$p$ value } \\
\hline & & & & $\begin{array}{l}\text { Lower } \\
\text { limit }\end{array}$ & $\begin{array}{l}\text { Upper } \\
\text { limit }\end{array}$ & \\
\hline $\begin{array}{l}\text { Number of medical } \\
\text { conditions/comorbidities }\end{array}$ & $>3$ vs. $\leq 3$ & 6.383 & 2.295 & 1.876 & 10.890 & $0.006^{*}$ \\
\hline
\end{tabular}

$C I$ Confidence interval, GP general practitioner, HDA Hellenic Diabetes Association, $N$ Number of subjects for whom information was available, $O L S$ ordinary least squares

*Significant difference at $p<0.05$
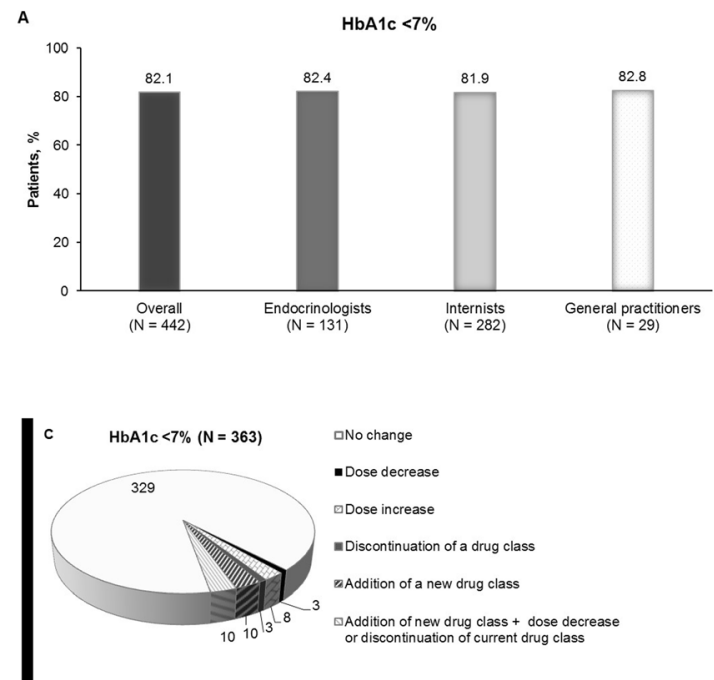

Fig. 4 Achievement of glycaemic control and action taken with current antidiabetic medication by physicians at enrolment. a Glycaemic control achievement rate, overall and according to physicians' specialty. Labels indicate the percentages of participants achieving control, and $N$ is the number of patients for whom data were available on haemoglobin $\mathrm{A}_{1 \mathrm{c}}\left(H b A_{I c}\right)$. b Glycaemic control achievement rate by number of antidiabetic treatment classes received. Labels indicate the percentages of participants

$\mathrm{HbA}_{1 \mathrm{c}}>9 \%$. The glycaemic control achievement rate according to the physicians' specialty is shown in Fig. 4a. The glycaemic control rate decreased as the number of OHAs increased, ranging from $91.6 \%$ in those receiving antidiabetic monotherapy to $58.8 \%$ in those receiving four OHAs (Fig. 4b).
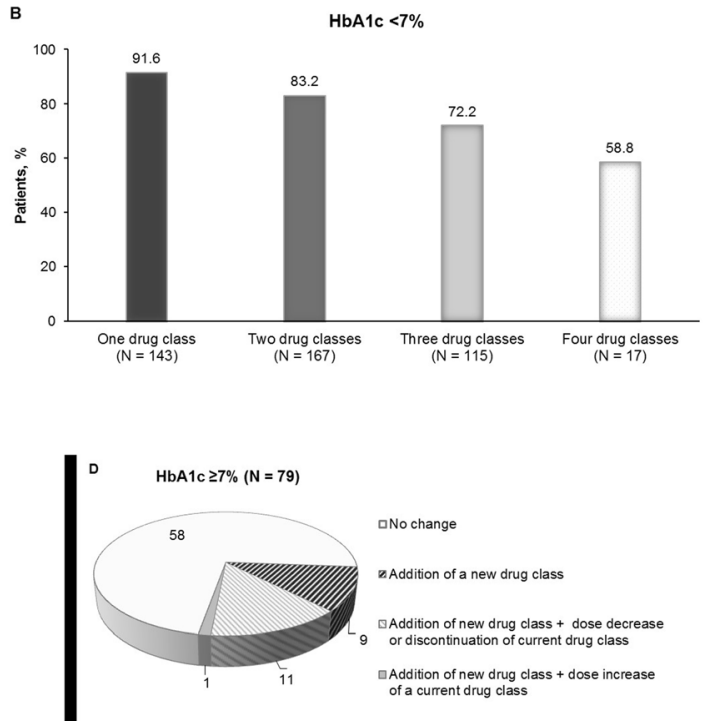

achieving control, and $N$ is the number of patients for whom data were available on $\mathrm{HbA}_{1 \mathrm{c}}$. c Distribution of participants [in absolute numbers $(N)$ ] attaining the target $\mathrm{HbA}_{1 \mathrm{c}}<7 \%$ according to the action to be taken with their antidiabetic medication at enrolment. $\mathbf{d}$ Distribution of participants [in absolute numbers $(N)$ ] failing to attain the target $\mathrm{HbA}_{1 \mathrm{c}}<7 \%$ according to the action to be taken with their antidiabetic medication at enrolment

\section{LDL-C, Blood Pressure, and Composite Metabolic Control at Enrolment}

Overall, the percentages of subjects achieving the LDL-C, SBP, DBP, composite SBP/DBP and composite metabolic targets were $57.0 \%(174 /$ 305) [with established CVD: 26.7\% (12/45), without CVD: 62.3\% (162/260)], 55.2\% (289/ 
524), $60.7 \%(318 / 524), 42.6 \%(223 / 524)[\geq 65$ years: $59.6 \%(159 / 267),<65$ years: $24.9 \%(64 /$ 257)] and $21.6 \%(58 / 268)$, respectively. The corresponding rates for SBP/DBP target achievement per physicians' specialty were $44.4 \%$ (59/133), $42.2 \%$ (152/360, and $38.7 \%$ $(12 / 31)$ for endocrinologists, internists and general practitioners respectively; achievement of the composite metabolic target was $21.6 \%$ (21/97), $21.5 \%(34 / 158)$ and $23.1 \%(3 / 13)$ for the aforementioned specialties.

\section{Changes in OHAs at Enrolment}

For $89.4 \%$ (537/601) of subjects, OHAs were not changed. Conversely, antidiabetic treatment was changed for 64 subjects (10.6\%), including 34 with $\mathrm{HbA}_{1 \mathrm{c}}<7 \%$, 21 with $\mathrm{HbA}_{1 \mathrm{c}} \geq 7 \%$ (Fig. 4c, d), and nine for whom $\mathrm{HbA}_{1 \mathrm{c}}$ data were unavailable. Changes in OHAs adhered to the HDA-recommended therapeutic algorithm in $83.5 \%(369 / 442)$ of subjects with available $\mathrm{HbA}_{1 \mathrm{c}}$ [in 96.7\% (351/363) of those with $\mathrm{HbA}_{1 \mathrm{c}}<7 \%$ and in $22.8 \%$ (18/79) of those with $\mathrm{HbA}_{1 \mathrm{c}} \geq 7 \%$ at enrolment].

\section{DISCUSSION}

The GLANCE study provides novel evidence on the adherence of Greek physicians to the PFP of the HDA guidelines in the routine care setting. It also provides data on the achievement rates for glycemic, blood pressure and lipid and composite metabolic control targets of non-insulin dependent subjects with T2DM in routine care settings.

The mean overall adherence of physicians to the PFP of the HDA guidelines was below 50\%. Physican adherence was highest for the laboratory evaluation domain (48\%), followed by the complete medical history domain (46\%) and then the physical examination domain (31\%). Slightly higher physician adherence rates were reported in the ADMIRE (Adherence of physicians to guidelines for the management of type 2 diabetes) study $[18,24]$, which had a similar design as the GLANCE study and evaluated physician adherence to the Turkish diabetes guidelines PFP in 1790 patients [18, 25]. The
Turkish and Greek PFP have several items in common, and the former separates the items into the same three domains as examined in the GLANCE study [20]. In ADMIRE, the mean overall adherence score for the 12 months prior to study enrolment was $70 \%$, with the scores (on 10-point scales) being 8.8 for the 5-item medical history domain, 6.3 for the 3 -item laboratory evaluation domain, and 5.9 for the 10-item physical examination domain [18]. The difference in the adherence scores between the two studies may be partly due to all 62 items of the HDA PFP being considered in the GLANCE study, whereas only 18 of the 39 items of the Turkish guidelines were evaluated in the ADMIRE study [18, 24, 25]. Additionally, the higher physician adherence rate in ADMIRE could, at least partly, be attributed some items of the PFP examined possibly being among those more commonly performed by physicians. For example, mean adherence score for the laboratory domain in the present study would be about $60 \%$ (and thus similar to the mean of 6.3 on a 10-point scale reported in ADMIRE), if it was based on the laboratory domain items included in ADMIRE [18, 24]. However, caution is needed when comparing the results of these two studies given that they differ in terms of patient characteristics. Importantly, in ADMIRE [24], 35\% of subjects received insulin, which was an exclusion criterion in the present study. Moreover, female sex was more frequent in ADMIRE (62\%) [24] than in the present study (45\%), and this has been shown to affect adherence, both in the present study and in a Luxembourg study [26]. Finally, in both the present study and in ADMIRE, diabetic complications and physicians' specialty were associated with adherence [18].

The results from the multivariable model on the factors affecting the overall PFP adherence require further investigation. For example, there are no specific cultural characteristics that may explain the increased adherence related to female subjects; and mixed findings have been reported in published data on the sex differences affecting the management of people with diabetes [27]. Furthermore, subjects with multiple comorbidities may have more regular access to the healthcare system and they also 
may receive more attention from their treating physician related to diabetes management and regular follow-up. In addition, the specialised and continuous education of endocrinologists and internal medicine physicians in diabetes could further provide a potential explanation of these results.

The general reasons reported for the poor adherence of physicians to guidelines include, among others, a lack of awareness or familiarity with the guidelines and/or the absence of any education on the use of the guidelines $[17,28,29]$. Indeed, in the ADMIRE study, overall physican adherence increased following the introduction of an education programme on the guidelines and diabetes complications [18]. Additional barriers to adherence may include poor healthcare finances and resources, inadequate healthcare system infrastructure and logistic support, excessive physician workload and the fact that physicians often doubt the utility of guidelines [29]. During the financial crisis in Greece, it was reported that prescriptions for OHAs were not affected [30], but decreased income and unemployment were identified as the major factors affecting access to healthcare services [31]. Specifically, the concerns of subjects with T2DM about treatment costs may affect their adherence to treatment and frequency of prescription fulfilment or even influence physicians' prescription behaviour towards choosing less expensive treatment options [32]. Moreover, budget cutbacks related to biochemical testing for and poor follow-up of diabetic complications that may prevent regular patient monitoring [32] could also play an important role in physician adherence to PFP. However, an inquiry into these factors was beyond the scope of GLANCE.

Of note, some studies have reported high adherence to guideline recommendations in terms of laboratory tests but inadequate glycemic control [33-35]. We found the reverse discrepancy, i.e. inadequate PFP adherence, but high glycaemic control (82.1\%). Impressively, this glycaemic control rate is the highest reported over the last decade in multi-centre studies in routine care Greek settings. In the recent AGREEMENT registry of 1191 adult patients with T2DM, adequate glycaemic control was achieved by $53 \%$ of subjects overall and by $65 \%$ of non-insulin-treated subjects [36]. Other studies have reported glycaemic control rates of $53-67 \%[15,30,37]$. In the GLANCE study, the LDL-C target was achieved by $57 \%$ of subjects, compared to 31\% [15] and 60\% [36] in previous Greek studies. In addition, adequate SBP control was achieved in the GLANCE study by $55 \%$ of subjects compared to a previously reported rate of $27 \%$ [15], while the composite metabolic control rate was $22 \%$ in this work compared to about 5\% in a previous study [15]. Arguably, our results point to some improvement-or at least some degree of stability-in the observed rates compared to those reported previously. Nonetheless, it should be mentioned that the present work was of a different design to those of the aforementioned Greek studies and had the primary aim to assess physician adherence to the HDA diabetes care guideline, with a secondary aim to capture the rates of achievement of glycemic, blood pressure and lipid targets.

Patient adherence to medication is known to affect the achievement of therapeutic targets $[38,39]$. In the present study, physicians reported that compliance to OHAs was very good/good in the vast majority (93\%) of subjects. Importantly, therapeutic decisions for changes in OHAs adhered to the HDA therapeutic algorithm in nearly nine of ten subjects. This high percentage probably reflects the wide acceptability of the HDA guidelines and the quality of the HDA-initiated physician education initiative on antidiabetic therapy.

Overall, the findings of GLANCE indicate that further efforts should focus on identifying potential barriers to the suboptimal adherence of physicians to the 62 PFP items and towards increasing physician awareness of the importance of comprehensive medical evaluation as outlined by HDA and international diabetes guidelines [20-23]. Moreover, given the available resources of physicians and taking into consideration potential financial and time constraints in routine care, it may be useful to take a closer look at the 62 items in the national PFP that were most met and least commonly met and potentially prioritise some items in the PFP. This could possibly optimise care for 
subjects with T2DM under the realistic situations encountered by physicians in daily practice [40]. On the other hand, the rapid evolution in digital solutions for diabetes management could further support patient followup protocols and improve outcomes [41]. However, as highlighted by the EASD and the ADA Diabetes Technology Working Group, several issues regarding the standardisation of diabetes digital applications should be addressed so that they can be validated in terms of their clinical significance and ensure patient safety and data privacy [42].

The strengths of the study include the large sample size and its multi-centric and nationwide nature. The recruitment of subjects from 10 of the 13 administrative regions of Greece (residence to $93.4 \%$ of the country's population) and the inclusion of endocrinologists, internists and general practitioners reflect variations in clinical practice. However, the study also has a number of limitations. First, bias in subject selection and information cannot be entirely ruled out. The former was minimised by consecutive enrolment. Regarding the latter, the rate of missing data on $\mathrm{HbA}_{1 \mathrm{c}}$, LDL-C and SBP/DBP measurements was between 13\% (SBP/ DBP) and 49\% (LDL-C). Moreover, data on diabetic complications were only collected from medical records; therefore, underestimation of their actual prevalence cannot be ruled out. A further limitation is that we did not use a central laboratory for measurements. Moreover, the inclusion of subjects with $\geq 2 \mathrm{HbA}_{1 \mathrm{c}}$ measurements may have contributed to overestimation of adherence. Also, the Likert scale used by physicians to evaluate patient compliance to antidiabetic treatment as well as patient adherence to physicians' recommendations represents per se a subjective rating method, which does not necessarily reflect the real situation. Lastly, results should be interpreted with caution, and a prospective study using all subjects with T2DM from the national diabetes registry could shed more light on current T2DM management in Greece.

\section{CONCLUSIONS}

The adherence of Greek physicians to the full PFP of the national diabetes guidelines is suboptimal. Of note, this adherence is affected by physicians' specialty as well as patient characteristics, such as sex, comorbidities and diabetic complications. Hence, future efforts should strive to identify potential barriers to guideline adherence in order to improve it and potentially prioritise the items included in the PFP, with the ultimate goal to optimally support subjects with T2DM.

\section{ACKNOWLEDGEMENTS}

Funding. This study and the journal's rapid service fee were funded by MSD, Greece.

Authors. All named authors meet the International Committee of Medical Journal Editors (ICMJE) criteria for authorship for this article, take responsibility for the integrity of the work as a whole, and have given their approval for this version to be published.

List of Investigators. The authors wish to thank the investigators in the GLANCE study: Agathi Vasileiou, Ekaterini Santipantaki, Ekaterini Trikkalinou, Antigoni Krasanaki, Antonios Antoniadis, Apostolos Xylomenos, Christos Manthakos, Chryssoula Gioti-Polyzou, Dimitra Zianni, Dimitrios Chrisis, Efrosini Tsirogianni, Eleni Pourou, Georgia Georgakopoulou, Georgia Katsigianni, Georgia Talampouka, Georgios Andreopoulos, Konstantinos Balampanis, Georgios Marinos, Haralampos Basoukos, Ilias Mazokopakis, Ioannis Giannakakis, Ioannis Katsakoulas, Iordanis Dimitsikoglou, Konstantinos Halkias, Konstantinos Lefkaditis, Kyros Siomos, Labrini Gkouva, Lazaros Chatziioannidis, Leonidas Peltekis, Louiza Ventzi, Maria Horianopoulou, Leonidas Lanaras, Nikolaos Valvis, Panagiotis Mylonas, Savvas Symeonidis, Spiridon Karras, Thekla Chatziadamidou, Vasileios Christakis, Vasileios Pikounis 
Disclosure. Nikolaos Papanas has been an advisory board member of TrigoCare International, Abbott, AstraZeneca, Elpen, MSD, Novartis, Novo Nordisk, Sanofi-Aventis and Takeda; has participated in sponsored studies by Eli Lilly, MSD, Novo Nordisk, Novartis and Sanofi-Aventis; received honoraria as a speaker for AstraZeneca, Boehringer Ingelheim, Eli Lilly, Elpen, Galenica, MSD, Mylan, Novartis, Novo Nordisk, Pfizer, Sanofi-Aventis, Takeda and Vianex; and attended conferences sponsored by TrigoCare International, AstraZeneca, Boehringer Ingelheim, Eli Lilly, Novartis, Novo Nordisk, Pfizer and Sanofi-Aventis. Moses Elisaf reports honoraria from MSD, Novartis, Chiesi, Bayer, Astra Zeneca, Pfizer, Abbott, Mylan, Sanofi, Amgen, Boehringer Ingelheim, Eli Lilly, GSK, Angelini, Winmedica, grants and personal fees from MSD and Astra Zeneca; has given talks and attended conferences sponsored by Bristol-Myers Squibb, Novartis, Chiesi, Bayer, Astra Zeneca, Pfizer, Abbott, Mylan, Sanofi, Amgen, Boehringer Ingelheim, Eli Lilly, Angelini, Winmedica and MSD. Kalliopi Kotsa has given talks, attended conferences, acted as a consultant and participated in trials sponsored by Astra Zeneca, Boehringer Ingelheim, Eli-Lilly, Elpen, MSD, Novo Nordisk, Sanofi and Servier. Alexandra Bargiota has received travel grands and/or participated in advisory boards for Astra-Zeneca, ELPEN, NovoNordisk,, MSD, Lilly, Novartis, Boehringer-Ingelheim and Sanofi-Aventis. John Doupis has been an advisory board member of, Abbott, AstraZeneca, Elpen, MSD, Novo Nordisk and Sanofi-Aventis; has participated in sponsored studies by Eli Lilly, MSD, Novo Nordisk, AstraZeneca Boehringer Ingelheim and SanofiAventis; received honoraria as a speaker for AstraZeneca, Boehringer Ingelheim, Eli Lilly, Elpen, MSD, Novo Nordisk, Sanofi-Aventis and Vianex; and attended conferences sponsored by AstraZeneca, MSD, Boehringer Ingelheim, Eli Lilly, Novo Nordisk and Sanofi-Aventis. Iakovos Avramidis has participated in advisory panels, speaker honoraria, clinical trials with: Sanofi Aventis, Eli Lilly, Novo Nordisk, Astra Zeneca, Boehringer Ingelheim, Novartis, MSD/Vianex, Elpen, GSK. Ioannis Ioannidise, Andreas Melidonis, Stavros Bousboulas, Angelos Pappas, Gerasimos Karousos, Eleni Arvaniti, Magdalini
Bristianou and Emmanouel Pagkalos report no potential conflicts of interest. Eugenia Karamousouli, Voss Bernd and Katerina Pietri are employees of Merck Sharp \& Dohme Corp., a subsidiary of Merck \& Co., Inc., Kenilworth, NJ, USA, who may own stock and/or hold stock options in the Company. Ilias Migdalis reports honoraria from MSD. Nikolaos Tentolouris has participated in advisory panels for MSD, AstraZeneca, Sanofi, Novo Nordisk, ELPEN, Eli Lilly, Boehringer Ingelheim and Novartis, and received research support from MSD, Eli Lilly, Novo Nordisk, Sanofi, Pfizer, AstraZeneca, Janssen Cilag, GSK, Trigocare, Elpen, Libytec and Novartis.

Compliance with Ethic Guidelines. The study was conducted in accordance with the International Society for Pharmacoepidemiology guidelines for Good Pharmacoepidemiology Practice, the ethical principles of the Declaration of Helsinki of 1964 and its later amendments and all standing regulations. The study was approved by the ethics committees of all participating hospitals (see Electronic Supplementary Material Table S1). Signed written informed consent was obtained from all participants.

Prior Presentation. Part of the manuscript was presented as an abstract in ISPOR Europe 2019 Congress, Copenhagen, Denmark.

Data Availability. The datasets generated and/or analysed during the current study are available from the corresponding author on reasonable request. MSD is committed to providing qualified scientific researchers access to anonymised patient-level data and clinical study reports from the company's clinical trials for the purpose of conducting legitimate scientific research. The company is also obliged to protect the rights and privacy of trial participants and, as such, has a procedure in place for evaluating and fulfilling requests for sharing company clinical trial data with qualified external scientific researchers. The process includes submission of data requests to the Merck data sharing website (available at http:// engagezone.merck.com/ds_documentation.php). 
There are circumstances that may prevent Merck from sharing the requested data.

Open Access. This article is licensed under a Creative Commons Attribution-NonCommercial 4.0 International License, which permits any non-commercial use, sharing, adaptation, distribution and reproduction in any medium or format, as long as you give appropriate credit to the original author(s) and the source, provide a link to the Creative Commons licence, and indicate if changes were made. The images or other third party material in this article are included in the article's Creative Commons licence, unless indicated otherwise in a credit line to the material. If material is not included in the article's Creative Commons licence and your intended use is not permitted by statutory regulation or exceeds the permitted use, you will need to obtain permission directly from the copyright holder. To view a copy of this licence, visit http://creativecommons.org/licenses/by$\mathrm{nc} / 4.0 /$.

\section{REFERENCES}

1. International Diabetes Federation (IDF). Atlas, 9th edition. Brussels: IDF. 2019. https://idf.org/ournetwork/regions-members/europe/members/137greece.html. Accessed 03 Mar 2020.

2. Liatis S, Dafoulas GE, Kani C, et al. The prevalence and treatment patterns of diabetes in the Greek population based on real-world data from the nation-wide prescription database. Diabetes Res Clin Pract. 2016;118:162-7.

3. International Diabetes Federation (IDF). Type 2 diabetes. Brussels: IDF. 2019. https://idf.org/52about-diabetes.html. Accessed 19 July 2019.

4. van der Leeuw J, Beulens JW, van Dieren S, et al. Novel biomarkers to improve the prediction of cardiovascular event risk in type 2 diabetes mellitus. J Am Heart Assoc. 2016;5:e003048.

5. Shah AD, Langenberg C, Rapsomaniki E, et al. Type 2 diabetes and incidence of cardiovascular diseases: a cohort study in 1.9 million people. Lancet Diabetes Endocrinol. 2015;3:105-13.

6. Cavender MA, Steg PG, Smith SC, et al. Impact of diabetes mellitus on hospitalization for heart failure, cardiovascular events, and death: Outcomes at 4 years from the reduction of atherothrombosis for continued health (REACH) registry. Circulation. 2015;132:923-31.

7. UK Prospective Diabetes Study Group. Tight blood pressure control and risk of macrovascular and microvascular complications in type 2 diabetes: UKPDS 38. BMJ. 1998;317:703-13.

8. Cholesterol Treatment Trialists' (CTT) Collaborators, Kearney PM, Blackwell L, et al. Efficacy of cholesterol-lowering therapy in 18,686 people with diabetes in 14 randomised trials of statins: a metaanalysis. Lancet. 2008;371:117-25.

9. American Diabetes Association (ADA). Chapter 10. Cardiovascular disease and risk management: standards of medical care in diabetes-2019. Diabetes Care. 2019;42(Suppl 1):S103-S123.

10. Stratton IM, Adler AI, Neil HA, et al. Association of glycaemia with macrovascular and microvascular complications of type 2 diabetes (UKPDS 35): prospective observational study. BMJ. 2000;321: 405-12.

11. Khunti K, Ceriello A, Cos X, De Block C. Achievement of guideline targets for blood pressure, lipid, and glycaemic control in type 2 diabetes: a metaanalysis. Diabetes Res Clin Pract. 2018;137:137-48.

12. Hermans MP, Brotons $\mathrm{C}$, Elisaf $\mathrm{M}$, et al. Optimal type 2 diabetes mellitus management: the randomised controlled OPTIMISE benchmarking study: baseline results from six European countries. Eur J Prev Cardiol. 2013;20:1095-105.

13. Tsimihodimos V, Bargiota A, Pagkalos EM, et al. Real-world adequacy of glycaemic control in treatment-naïve Greek patients with type 2 diabetes mellitus initiating treatment with metformin monotherapy at the maximum tolerated dose: the Reload study. Exp Clin Endocrinol Diabetes. 128(4): 224-30. https://doi.org/10.1055/a-0824-6607.

14. Pagkalos E, Thanopoulou A, Sampanis C, et al. The real-life effectiveness and care patterns of type 2 diabetes management in Greece. Exp Clin Endocrinol Diabetes. 2018;126:53-60.

15. Kostapanos MS, Tsimihodimos V, Elisaf MS, Tzouvelekis E, Nikas N. Rationale, design and baseline patient characteristics of the optimal type 2 diabetes management including benchmarking and standard treatment study in Greece. World J Diabetes. $2014 ; 5: 76-83$.

16. Tsimihodimos V, Kostapanos MS, Moulis A, Nikas $\mathrm{N}$, Elisaf MS. Effects of benchmarking on the quality of type 2 diabetes care: results of the OPTIMISE (Optimal Type 2 Diabetes Management Including 
Benchmarking and Standard Treatment) study in Greece. Ther Adv Endocrinol Metab. 2015;6: 199-209.

17. Cabana MD, Rand CS, Powe NR, et al. Why don't physicians follow clinical practice guidelines? A framework for improvement. JAMA. 1999;282: 1458-65.

18. Satman I, Imamoglu S, Yilmaz C, ADMIRE Study Group. Adherence to guidelines and its effect on glycemic control during the management of type 2 diabetes in Turkey: The ADMIRE Study. In: Sameh Magdeldin, editor, State of the art of therapeutic endocrinology. London: IntechOpen. 2012. https:// www.intechopen.com/books/state-of-the-art-oftherapeutic-endocrinology/adherence-toguidelines-and-its-effect-on-glycemic-controlduring-the-management-of-type-2-diabetes. Accessed 22 July 2019.

19. Oude Wesselink SF, Lingsma HF, Robben PB, Mackenbach JP. Guideline adherence and health outcomes in diabetes mellitus type 2 patients: a cross-sectional study. BMC Health Serv Res. 2015;15:22.

20. Hellenic Diabetes Association (HDA). Guidelines for the management of the diabetic patient, 2017. Athens: HDA. 2017. https://www.ede.gr/wpcontent/uploads/2017/odigies.pdf. Accessed 31 Oct 2017.

21. Inzucchi SE, Bergenstal RM, Buse JB, et al. Management of hyperglycemia in type 2 diabetes, 2015: a patient-centered approach: update to a position statement of the American Diabetes Association and the European Association for the Study of Diabetes. Diabetes Care. 2015;38:140-9.

22. American Diabetes Association (ADA). Standards of medical care in diabetes 2017. Chapter6: glycemic targets. Diabetes Care. 2017;40(Suppl. 1):S48-S56.

23. American Diabetes Association (ADA). Standards of medical care in diabetes 2017. Chapter 9: cardiovascular disease and risk management. Diabetes Care. 2017;40(Suppl 1):S75-S87.

24. Satman I, Imamoglu S, Yilmaz C, ADMIRE Study Group. A patient-based study on the adherence of physicians to guidelines for the management of type 2 diabetes in Turkey. Diabetes Res Clin Pract. 2012;98:75-82.

25. Satman I, Imamoglu S, Yilmaz C, SEMT Diabetes Study Group (Editors). Clinical practice guidelines for diagnosis, treatment, and follow-up of diabetes mellitus and its complications. Updated Fourth Edition. Turk Jem. 2010;14. ISSN: 1301-2193 E-ISSN: 1308-9846.
26. Renard LM, Bocquet V, Vidal-Trecan G, Lair ML, Blum-Boisgard C. Adherence to international follow-up guidelines in type 2 diabetes: a longitudinal cohort study in Luxembourg. PLoS ONE. 2013;8: e80162.

27. de Jong M, Vos RC, de Ritter R, et al. Sex differences in cardiovascular risk management for people with diabetes in primary care: a cross-sectional study. Open. 2019;3(2):bjgpopen19X101645. https://doi. org/10.3399/bjgpopen19X101645.

28. Fürthauer J, Flamm M, Sönnichsen A. Patient and physician related factors of adherence to evidence based guidelines in diabetes mellitus type 2, cardiovascular disease and prevention: a cross sectional study. BMC Fam Pract. 2013;14:47.

29. Hashmi NR, Khan SA. Adherence to diabetes mellitus treatment guidelines from theory to practice: the missing link. J Ayub Med Coll Abbottabad. 2016;28:802-8.

30. Liatis S, Papaoikonomou S, Ganotopoulou A, et al. Management of type 2 diabetes and its prescription drug cost before and during the economic crisis in Greece: an observational study. BMC Endocrine Disorders. 2014;14:23.

31. Kyriopoulos I, Zavras D, Skroumpelos A, et al. Barriers in access to healthcare services for chronic patients in times of austerity: an empirical approach in Greece. Int J Equity Health. 2014;13: 54.

32. Aloumanis K, Papanas N. Greek financial crisis: consequences in the healthcare of diabetes and its complications. Hippokratia. 2014;18:4-6.

33. Coon P, Zulkowski K. Adherence to American Diabetes Association standards of care by rural health care providers. Diabetes Care. 2002;25:2224-49.

34. Oh SW, Lee HJ, Chin HJ, Hwang JI. Adherence to clinical practice guidelines and outcomes in diabetic patients. Int J Qual Health Care. 2011;23: 413-9.

35. Rao DT, Sunio LK, Lo YJ, Gossain VV. Comparison of the adherence to the American Diabetes Association guidelines of diabetes care in primary care and subspecialty clinics. J Diabetes Metab Disord. $2015 ; 14: 35$.

36. Liatis S, Iraklianou S, Kazakos K, et al. A Greek registry of current type 2 diabetes management, aiming to determine core clinical approaches, patterns and strategies. BMC Endocr Disord. 2019;19: 39.

37. de Pablos-Velasco P, Parhofer KG, Bradley C, et al. Current level of glycaemic control and its 
associated factors in patients with type 2 diabetes across Europe: data from the PANORAMA study. Clin Endocrinol (Oxf). 2014;80:47-56.

38. Ahmad NS, Ramli A, Islahudin F, Paraidathathu T. Medication adherence in patients with type 2 diabetes mellitus treated at primary health clinics in Malaysia. Patient Prefer Adherence. 2013;7:525-30.

39. Rezaei M, Valiee S, Tahan M, Ebtekar F, Ghanei GR. Barriers of medication adherence in patients with type-2 diabetes: a pilot qualitative study. Diabetes Metab Syndr Obes. 2019;12:589-99.

40. American Diabetes Association (ADA). 4. Comprehensive medical evaluation and assessment of comorbidities: standards of medical care in diabetes-2019. Diabetes Care. 2019;42(Suppl 1): S34-S45.

41. Doupis J, Festas G, Tsilivigos C, Efthymiou E, Kokkinos A. Smartphone-based technology in diabetes management. Diabetes Ther. 2020;11:607-19.

42. Fleming G, Petrie J, Bergenstal R, et al. Diabetes digital app technology: benefits, challenges, and recommendations. A consensus report by the European Association for the Study of Diabetes (EASD) and the American Diabetes Association (ADA) Diabetes Technology Working Group. Diabetes Care. 2019;4:250-60. 